\title{
Error Modeling, Calibration, and Nonlinear Interpolation Compensation Method of Ring Laser Gyroscope Inertial Navigation System
}

\author{
Jianli Li, ${ }^{1,2}$ Yanhai Ma, ${ }^{1,2}$ and Chen Xiyuan ${ }^{3}$ \\ ${ }^{1}$ Fundamental Science on Novel Inertial Instrument \& Navigation System Technology Laboratory, School of Instrumentation Science \\ $\&$ Optoelectronics Engineering, Beijing University of Aeronautics and Astronautics, Beijing 100191, China \\ ${ }^{2}$ Science and Technology on Inertial Laboratory, Beijing 100191, China \\ ${ }^{3}$ School of Instrumentation Science \& Engineering, Southeast University, Nanjing 211100, China
}

Correspondence should be addressed to Jianli Li; iilijianli@yahoo.com.cn

Received 25 November 2012; Revised 30 January 2013; Accepted 18 February 2013

Academic Editor: Chuandong Li

Copyright (C) 2013 Jianli Li et al. This is an open access article distributed under the Creative Commons Attribution License, which permits unrestricted use, distribution, and reproduction in any medium, provided the original work is properly cited.

In order to improve the precision of Strapdown Inertial Navigation System (SINS) and reduce the complexity of the traditional calibration method, a novel calibration and compensation scheme is proposed. An optimization calibration method with fourdirection rotations is designed to calculate all error coefficients of Ring Laser Gyroscope (RLG) SINS in a series of constant temperatures. According to the actual working environment, the temperature errors of RLG SINS are compensated by a nonlinear interpolation compensation algorithm. The experimental results show that the inertial navigation errors of the proposed method are reduced.

\section{Introduction}

Motion error compensation based on a high precision Position and Orientation System (POS) is an effective avenue to improve the imaging quality of the interferometric Synthetic Aperture Radar (SAR) [1, 2]. The Strapdown Inertial Navigation System (SINS) of the POS is fixed on the antenna of the SAR outside the aircraft cabin; so, the measurement results are easily affected by the extreme temperature environment. Therefore, an accurate calibration and compensation method for SINS is necessary to improve the measurement precision and environmental adaptability of SINS [3].

The core sensors of SINS are the gyroscopes and accelerometers; the gyroscopes are sensitive to the rotation rate, and the accelerometers are sensitive to the acceleration. So, the precision of SINS depends on the precision of the gyroscopes and accelerometers. The traditional calibration methods for SINS include static multiposition calibration method [4], rotation rate calibration method [5], and dynamic-static hybrid calibration method [6], and so on. Among all the methods, the static multiposition calibration method can accurately calibrate the error coefficients of acceleration channel, while the accuracy of the error coefficients in angular rate channel is very low. On the other hand, the traditional rotation rate calibration can improve the precision of the angular rate channel error coefficients, but it reduces the precision of acceleration channel. Reference [7] proposed a 24-point dynamic-static hybrid calibration method. The method improves the precision of the calibrated coefficients in both of the two channels, but increasing the complexity of the calibration. So, it is another key technology for SINS to find a better method to reduce the complexity and error of the calibration at the same time.

Furthermore, the change of the working environmental temperature also decreases the precision of the SINS significantly $[8,9]$. The linear fitting method is often applied to compensate for the temperature errors of inertial devices biases and scale factors. The method is easy to be implemented, while the precision of compensation is low. Other nonlinear methods, such as the high order least-squares approximation method and the Radial Basis Function (RBF) neural network method, improve the precision of the SINS, but they cannot 
meet the requirement of real-time processing because of the large amount of calculation. So, it is another key problem for SINS to resolve the previous conflicts. Based on the theoretical analysis and experimental data, the calibration and compensation methods of the temperature and dynamic error for SINS are studied in this paper, and an optimization calibration method with four-direction rotations and nonlinear interpolation compensation method is proposed for SINS in full temperature ranges.

\section{Error Modeling of RLG SINS}

2.1. Error Modeling of the Angular Rate Channel. Dithered Ring Laser Gyroscope (RLG) is not sensitive to the gravity acceleration. Considering the scale factors, biases, and misalignment errors, the error model of angular rate channel is written as

$$
\left[\begin{array}{l}
G_{x} \\
G_{y} \\
G_{z}
\end{array}\right]=\left[\begin{array}{l}
G_{b x} \\
G_{b y} \\
G_{b z}
\end{array}\right]+\left[\begin{array}{lll}
S_{g x} & E_{x y} & E_{x z} \\
E_{y x} & S_{g y} & E_{y z} \\
E_{z x} & E_{z y} & S_{g z}
\end{array}\right]\left[\begin{array}{c}
\omega_{x} \\
\omega_{y} \\
\omega_{z}
\end{array}\right],
$$

where $G_{i}$ and $G_{b i}$ are gyroscope output and bias in $i$ axis, $\omega_{i}$ is inputting rational rate, $S_{g i}$ is gyroscope scale factor, and $E_{i j}$ is the misalignment error of angular rate channel, where $i=$ $x, y, z ; j=x, y, z$.

2.2. Error Modeling of the Acceleration Channel. According to the characteristic of the Quartz Mechanical Accelerometer (QMA), the acceleration channel error model of the SINS can be given by

$$
\left[\begin{array}{l}
A_{x} \\
A_{y} \\
A_{z}
\end{array}\right]=\left[\begin{array}{l}
A_{b x} \\
A_{b y} \\
A_{b z}
\end{array}\right]+\left[\begin{array}{ccc}
S_{a x} & M_{x y} & M_{x z} \\
M_{y x} & S_{a y} & M_{y z} \\
M_{z x} & M_{z y} & S_{a z}
\end{array}\right]\left[\begin{array}{c}
a_{x} \\
a_{y} \\
a_{z}
\end{array}\right],
$$

where $A_{i}$ is the accelerometer output of RLG SINS in $i$ axis, $a_{i}$ is the accelerometer input, $A_{b i}$ is the accelerometer bias, $S_{a i}$ is the acceleration scale factor, and $M_{i j}$ is the misalignment error of the acceleration channel, where $i=x, y, z ; j=x, y, z$.

\section{The Optimization Calibration Method with Four-Direction Rotations}

The measurement precision of RLG SINS is mostly affected by the temperature variation, the change rate, and the gradient of the temperature. The work temperature of airborne SAR varies a lot with the flight altitude. Compared with the temperature variation, the gradient and change rate of the temperature do not change much during every procession of airborne imaging. The effects of different temperature on the precision of RLG SINS are mainly studied in this paper. The range of calibration temperature can be set according to the actual working environment and the accuracy requirement.

According to the error model of RLG SINS, an optimization calibration method with four-direction rotations is designed for RLG SINS based on a single-axis turntable with temperature control and a high-precision hexahedral fixture. In the course of calibration, the SINS fixed in hexahedral fixture will be overturn four times at each temperature point, making the $X, Y$, and $Z$ axes of SINS superposed with $Z_{t}$ axis and $-Z_{t}$ axis of the turntable $\left(Z_{t}\right.$ axis points up in Geographic Coordinate System). At each direction, the turntable will be pivoted the angle of $2 \pi n$ with $n$ being the cycle number. When $x$-axis directs up as the first direction as shown in Figure 1, the inputting angular rates of the three sensing axes can be given by

$$
\left[\begin{array}{c}
\omega_{x}(t) \\
\omega_{y}(t) \\
\omega_{z}(t)
\end{array}\right]=\left[\begin{array}{c}
\omega_{i e} \cos \phi \sin \varphi(t) \\
\omega_{i e} \cos \phi \cos \varphi(t) \\
\Omega+\omega_{i e} \sin \phi
\end{array}\right]
$$

where $\omega_{i e}$ is rotation rate of earth. $\phi$ is local latitude. $\Omega$ is the inputting rotation rate of turntable. $\varphi(t)$ is head of RLG IMU. The inputting acceleration of the three sensing axes can be given by

$$
\left[\begin{array}{l}
a_{x}(t) \\
a_{y}(t) \\
a_{z}(t)
\end{array}\right]=\left[\begin{array}{l}
0 \\
0 \\
g
\end{array}\right]
$$

The mean value of inputting angular rates and acceleration at the first direction can be obtained as

$$
\begin{gathered}
{\left[\begin{array}{l}
\omega_{x} \\
\omega_{y} \\
\omega_{z}
\end{array}\right]=\frac{\Omega}{2 \pi n}\left[\begin{array}{l}
\int_{0}^{2 \pi n / \Omega} \omega_{x}(t) d t \\
\int_{0}^{2 \pi n / \Omega} \omega_{y}(t) d t \\
\int_{0}^{2 \pi n / \Omega} \omega_{z}(t) d t
\end{array}\right]} \\
=\left[\begin{array}{c}
0 \\
0 \\
\Omega+\omega_{i e} \sin \phi
\end{array}\right]=\left[\begin{array}{l}
0 \\
0 \\
\omega
\end{array}\right], \\
{\left[\begin{array}{l}
a_{x} \\
a_{y} \\
a_{z}
\end{array}\right]=\frac{\Omega}{2 \pi n}\left[\begin{array}{l}
\int_{0}^{2 \pi n / \Omega} a_{x}(t) d t \\
\int_{0}^{2 \pi n / \Omega} a_{y}(t) d t \\
\int_{0}^{2 \pi n / \Omega} a_{z}(t) d t
\end{array}\right]=\left[\begin{array}{l}
0 \\
0 \\
g
\end{array}\right] .}
\end{gathered}
$$

The system states equation of angular rate channels of RLG SINS during the optimization calibration can be expressed as

$$
\left[\begin{array}{ccc}
G_{x 1} & G_{y 1} & G_{z 1} \\
G_{x 2} & G_{y 2} & G_{z 2} \\
G_{x 3} & G_{y 3} & G_{z 3} \\
G_{x 4} & G_{y 4} & G_{z 4}
\end{array}\right]=\left[\begin{array}{cccc}
1 & \bar{\omega} & 0 & 0 \\
1 & 0 & \bar{\omega} & 0 \\
1 & 0 & 0 & \bar{\omega} \\
1 & 0 & 0 & -\bar{\omega}
\end{array}\right]\left[\begin{array}{ccc}
G_{b x} & G_{b y} & G_{b z} \\
S_{g x} & E_{y x} & E_{z x} \\
E_{x y} & S_{g y} & E_{z y} \\
E_{x z} & E_{y z} & S_{g z}
\end{array}\right],
$$

where $\bar{\omega}$ is inputting angular rate; $\bar{\omega}=\Omega+\omega_{i e} \sin \phi . G_{i m}$ is gyroscope output in $i$ axis for the $m$ th direction, where 


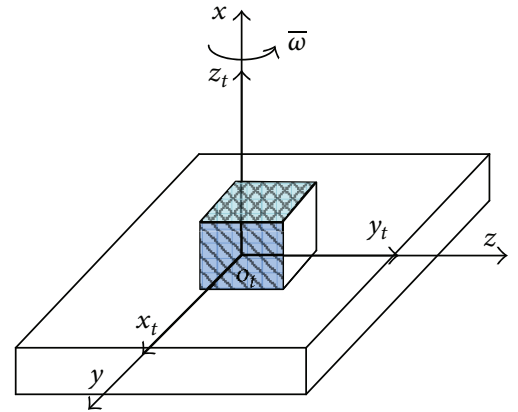

(1) The first direction

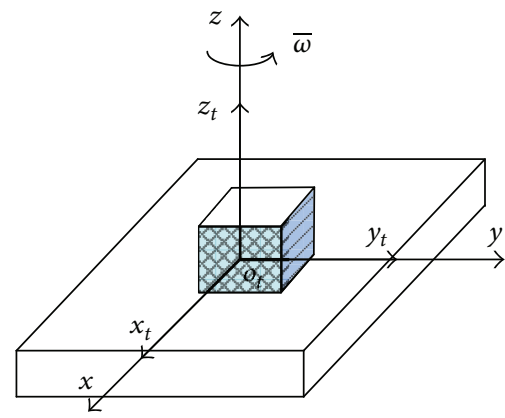

(3) The third direction

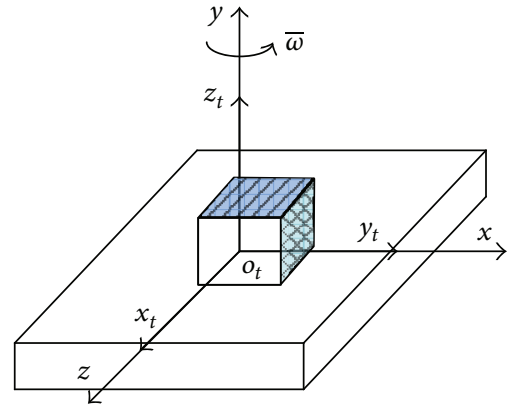

(2) The second direction

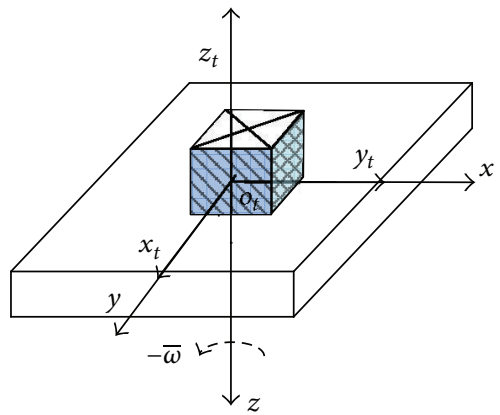

(4) The fourth direction

FIGURE 1: An optimization four-direction rotation rate calibration method using a single-axis turntable with temperature control.

$i=x, y, z ; m=1,2,3,4$. The system status equation of acceleration channel of RLG SINS can be given by

$$
\left[\begin{array}{ccc}
A_{x 1} & A_{y 1} & A_{z 1} \\
A_{x 2} & A_{y 2} & A_{z 2} \\
A_{x 3} & A_{y 3} & A_{z 3} \\
A_{x 4} & A_{y 4} & A_{z 4}
\end{array}\right]=\left[\begin{array}{cccc}
1 & g & 0 & 0 \\
1 & 0 & g & 0 \\
1 & 0 & 0 & g \\
1 & 0 & 0 & -g
\end{array}\right]\left[\begin{array}{ccc}
A_{b x} & A_{b y} & A_{b z} \\
S_{a x} & M_{y x} & M_{z x} \\
M_{x y} & S_{a y} & M_{z y} \\
M_{x z} & M_{y z} & S_{a z}
\end{array}\right],
$$

where $A_{\text {im }}$ is the output of the $i$ axis accelerometer on the $m$ th direction. According to (6) and (7), the error coefficients of angular rate and acceleration channels of RLG SINS can be calculated by

$$
\begin{aligned}
& {\left[\begin{array}{ccc}
G_{b x} & G_{b y} & G_{b z} \\
S_{g x} & E_{y x} & E_{z x} \\
E_{x y} & S_{g y} & E_{z y} \\
E_{x z} & E_{y z} & S_{g z}
\end{array}\right]} \\
& =\frac{1}{2 \bar{\omega}}\left[\begin{array}{ccc}
\bar{\omega}\left(G_{x 3}+G_{x 4}\right) & \bar{\omega}\left(G_{y 3}+G_{y 4}\right) & \bar{\omega}\left(G_{z 3}+G_{z 4}\right) \\
2\left(G_{x 1}-G_{b x}\right) & 2\left(G_{y 1}-G_{b x}\right) & 2\left(G_{z 1}-G_{b x}\right) \\
2\left(G_{x 2}-G_{b x}\right) & 2\left(G_{y 2}-G_{b x}\right) & 2\left(G_{z 2}-G_{b x}\right) \\
G_{x 3}-G_{x 4} & G_{y 3}-G_{y 4} & G_{z 3}-G_{z 4}
\end{array}\right],
\end{aligned}
$$

$$
\begin{aligned}
& {\left[\begin{array}{ccc}
A_{b x} & A_{b y} & A_{b z} \\
S_{a x} & M_{y x} & M_{z x} \\
M_{x y} & S_{a y} & M_{z y} \\
M_{x z} & M_{y z} & S_{a z}
\end{array}\right]} \\
& =\frac{1}{2 g}\left[\begin{array}{ccc}
g\left(A_{x 3}+A_{x 4}\right) & g\left(A_{y 3}+A_{y 4}\right) & g\left(A_{z 3}+A_{z 4}\right) \\
2\left(A_{x 1}-A_{b x}\right) & 2\left(A_{y 1}-A_{b y}\right) & 2\left(A_{z 1}-A_{b z}\right) \\
2\left(A_{x 2}-A_{b x}\right) & 2\left(A_{y 2}-A_{b y}\right) & 2\left(A_{z 2}-A_{b z}\right) \\
A_{x 3}-A_{x 4} & A_{y 3}-A_{y 4} & A_{z 3}-A_{z 4}
\end{array}\right] .
\end{aligned}
$$

\section{Nonlinear Interpolation Compensation of Temperature Errors}

Both the RLG and QMA are sensitive to the temperature, and the precision of RLG SINS is limited by the temperature errors. Two measures are usually taken to solve the problem: (1) adopting temperature control technology to reduce the change of temperature [10]; (2) establishing the mathematical model of the temperature errors based on the analysis of the test data and using compensation algorithm to reduce the temperature errors [11]. The first method is disadvantageous in the high cost and an increase of the size and weight of RLG SINS. Due to the easy implementation and validity, the second method draws lots of the researcher's attention. The second method is implemented by analyzing the test 


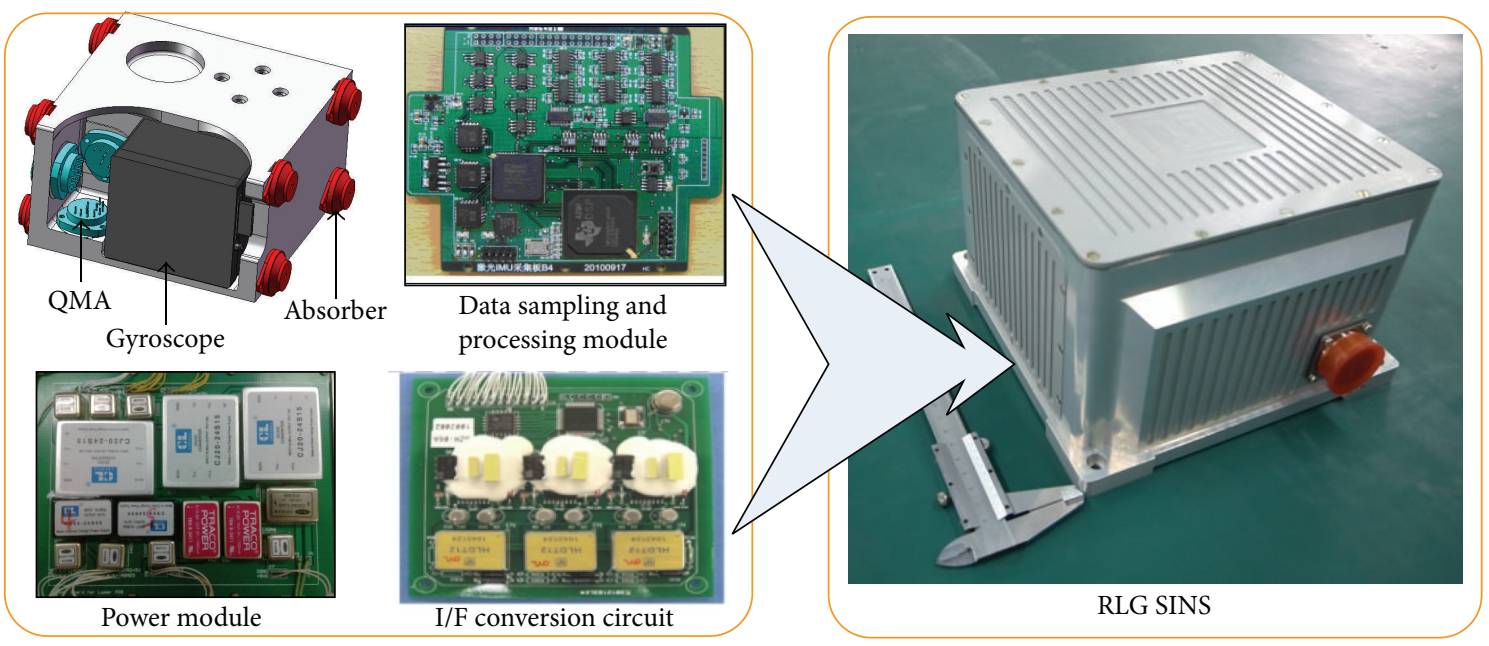

FIGURE 2: The hardware components and operation principle of RLG POS.

data sampled by the RLG SINS to find the hidden relation between the outputs of RLG SINS and the external temperature.

The conventional methods of the errors compensation include the linear fitting method and RBF neural network method. The linear fitting method is commonly used to find the hidden law of data. Though the method has the least amount of calculation, it does not meet the precision demand. Other nonlinear approximation methods, such as the high order least-squares approximation method and the RBF neural network method, can improve the precision of the SINS, but they increase the amount of calculation. Due to the great complexity of the algorithm, these methods are not suitable for real-time processing in RLG POS.

Considering the precision and calculational amount, a nonlinear low-order interpolation method is used to compensate for the temperature errors of RLG SINS. The method divides the temperature into several small intervals and uses piecewise linear interpolation in each interval. Due to the closed-loop control of RLG, the error of scale factor is very small. The scale factor temperature errors of RLG SINS are unnecessary to be compensated. A series of calibration temperature points are supposed as $T=$ $\left[\begin{array}{llllll}T_{1} & T_{2} & \cdots & T_{k} & \cdots & T_{n}\end{array}\right]$. If the working temperature is $T \in$ $\left[T_{k-1}, T_{k}\right](k=1,2, \ldots, n)$, the piecewise linear interpolation algorithm about the temperature errors of RLG biases can be expressed as follows:

$$
\left[\begin{array}{l}
G_{b x}(T) \\
G_{b y}(T) \\
G_{b z}(T)
\end{array}\right]=\left[\begin{array}{l}
\frac{T-T_{k}}{T_{k-1}-T_{k}} G_{b x, k-1}+\frac{T-T_{k-1}}{T_{k}-T_{k-1}} G_{b x, k} \\
\frac{T-T_{k}}{T_{k-1}-T_{k}} G_{b y, k-1}+\frac{T-T_{k-1}}{T_{k}-T_{k-1}} G_{b y, k} \\
\frac{T-T_{k}}{T_{k-1}-T_{k}} G_{b z, k-1}+\frac{T-T_{k-1}}{T_{k}-T_{k-1}} G_{b z, k}
\end{array}\right]
$$

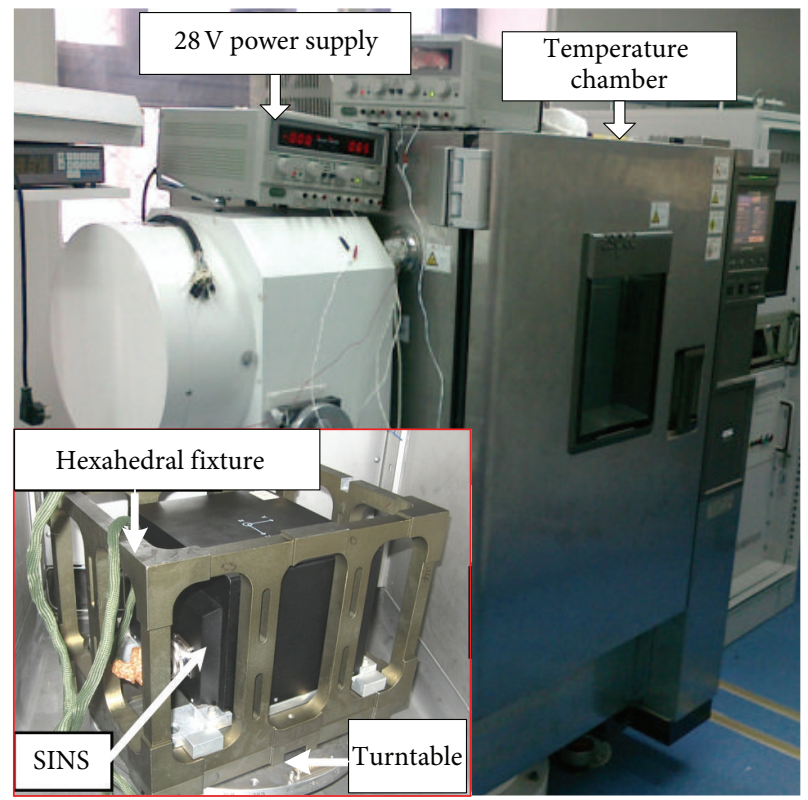

FIgURE 3: The single-axis turntable with temperature control for the calibration experiment.

The temperature errors of accelerometer biases and scale factors are given by

$$
\left[\begin{array}{l}
A_{b x}(T) \\
A_{b y}(T) \\
A_{b z}(T)
\end{array}\right]=\left[\begin{array}{l}
\frac{T-T_{k}}{T_{k-1}-T_{k}} A_{b x, k-1}+\frac{T-T_{k-1}}{T_{k}-T_{k-1}} A_{b x, k} \\
\frac{T-T_{k}}{T_{k-1}-T_{k}} A_{b y, k-1}+\frac{T-T_{k-1}}{T_{k}-T_{k-1}} A_{b y, k} \\
\frac{T-T_{k}}{T_{k-1}-T_{k}} A_{b z, k-1}+\frac{T-T_{k-1}}{T_{k}-T_{k-1}} A_{b z, k}
\end{array}\right]
$$


TABLE 1: The 3600 seconds position errors of inertial navigation in vehicle experiment.

\begin{tabular}{lcccc}
\hline Experiment & Method & Latitude/nautical mile & Longitude/nautical mile & Position/nautical mile \\
\hline Vehicle experiment & $\begin{array}{c}\text { 24-point hybrid calibration and linear } \\
\text { fitting method } \\
\text { Proposed method }\end{array}$ & 1.841 & 0.183 & 1.850 \\
& $\begin{array}{c}\text { Pros } \\
\text { V }\end{array}$ & 0.408 & 0.431 & 0.594 \\
\hline
\end{tabular}
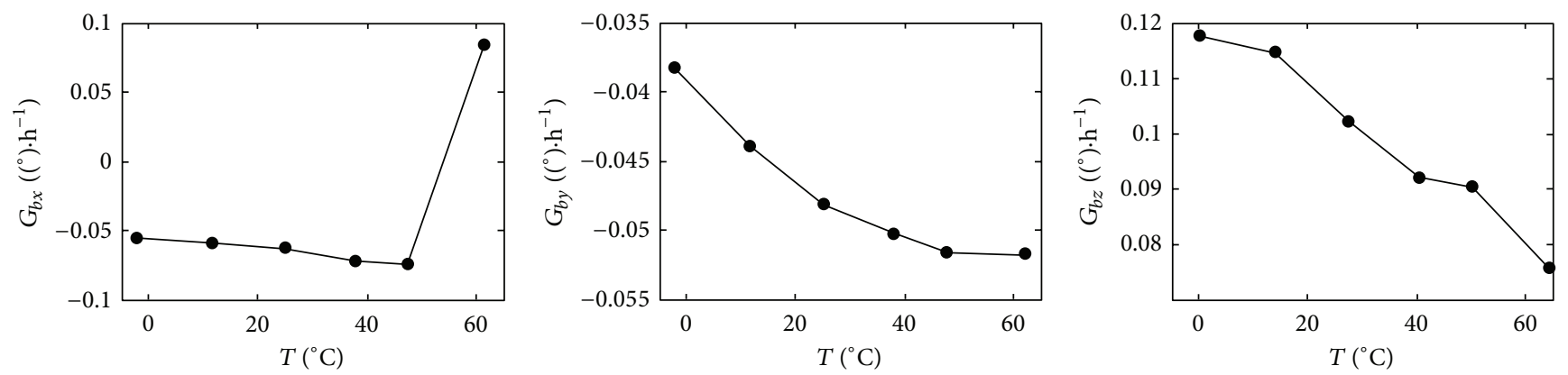

Figure 4: Curves of gyroscope bias changing with temperature.

$$
\left[\begin{array}{l}
S_{a x}(T) \\
S_{a y}(T) \\
S_{a Z}(T)
\end{array}\right]=\left[\begin{array}{l}
\frac{T-T_{k}}{T_{k-1}-T_{k}} S_{a x, k-1}+\frac{T-T_{k-1}}{T_{k}-T_{k-1}} S_{a x, k} \\
\frac{T-T_{k}}{T_{k-1}-T_{k}} S_{a y, k-1}+\frac{T-T_{k-1}}{T_{k}-T_{k-1}} S_{a y, k} \\
\frac{T-T_{k}}{T_{k-1}-T_{k}} S_{a z, k-1}+\frac{T-T_{k-1}}{T_{k}-T_{k-1}} S_{a z, k}
\end{array}\right] .
$$

\section{Experiment and Result}

5.1. Calibration Experiment of RLG SINS. The RLG SINS hardware includes an Inertial Sensing Assembly (ISA), a power module, eight absorbers, an I/F conversion circuit, and a data sampling and processing module as shown in Figure 2. The ISA includes three RLGs and three QMAs assembled in a supporting structure. The data sampling and processing module completes the data sampling from the RLG SINS, data filtering, synchronization, errors compensation, and real-time inertial navigation arithmetic. The sampling data are sent to the integrated navigation computer by RS422 serial port, and the Kalman filter is used in SINS/ Global Position System (GPS) integrated navigation. The integrated navigation data are sent to airborne SAR to realize real-time motion error compensation. In addition, the original data of SINS and GPS can be restored for postprocessing to improve the precision and smoothness of integrated navigation results and get a higher image resolution of SAR.

According to the actual working environment and precision requirement of RLG POS, the calibration experiment is carried on in the temperature points of $-25^{\circ} \mathrm{C},-10^{\circ} \mathrm{C}$, $5^{\circ} \mathrm{C}, 20^{\circ} \mathrm{C}, 30^{\circ} \mathrm{C}$, and $40^{\circ} \mathrm{C}$. The RLG SINS is fixed in the center of the hexahedral fixture, and the $x, y$, and $z$ axes are perpendicular to the datum plane of the turntable separately as shown in Figure 1. The calibration experiment is implemented by turning over the hexahedral fixture, which is placed on the single-axis turntable with temperature control as shown in Figure 3.

According to (6)-(8), the error coefficients of acceleration and angular rate channels can be calibrated. The curves of gyroscope bias changing with temperature are shown in Figure 4, and the curves of accelerometer biases and scale factors changing with temperature are shown in Figure 5. Compared to the traditional 24-point hybrid calibration method without regard to temperature effect, the calibration time of the proposed method is declining from about 3 hours to 1.5 hours. There is no necessity to calibrate the temperature transducers. Since the heating of POS, the temperatures of transducers are higher than the environmental temperature as shown in Figures 4 and 5. The gyroscope biases, acceleration biases, and scale factor are nonlinear with respect to the temperature.

5.2. Performance Test of RLG SINS. In order to validate the method proposed in this paper, the proposed method and the traditional 24-point dynamic-static hybrid calibration method are used to calibrate the coefficients separately, and then vehicle experiments and airborne experiments are conducted. The RLG POS was fixed on an immovability marble platform in the experimental vehicle. The GPS antenna was fitted on the top of the vehicle, and the GPS receiver was placed in the vehicle. The POS power was switched on. Firstly, the vehicle was parked in a location for 300 seconds, and the data were sampled for initial alignment. Then, the vehicle was driven along layout route for more than 3600 seconds. Two groups of tests were conducted with each method.

Comparing the vehicle experiment navigation results with the GPS positioning results, the precisions of inertial navigation in the two methods are shown in Table 1. The experimental results show that the inertial navigation average error of the proposed method is reduced to 

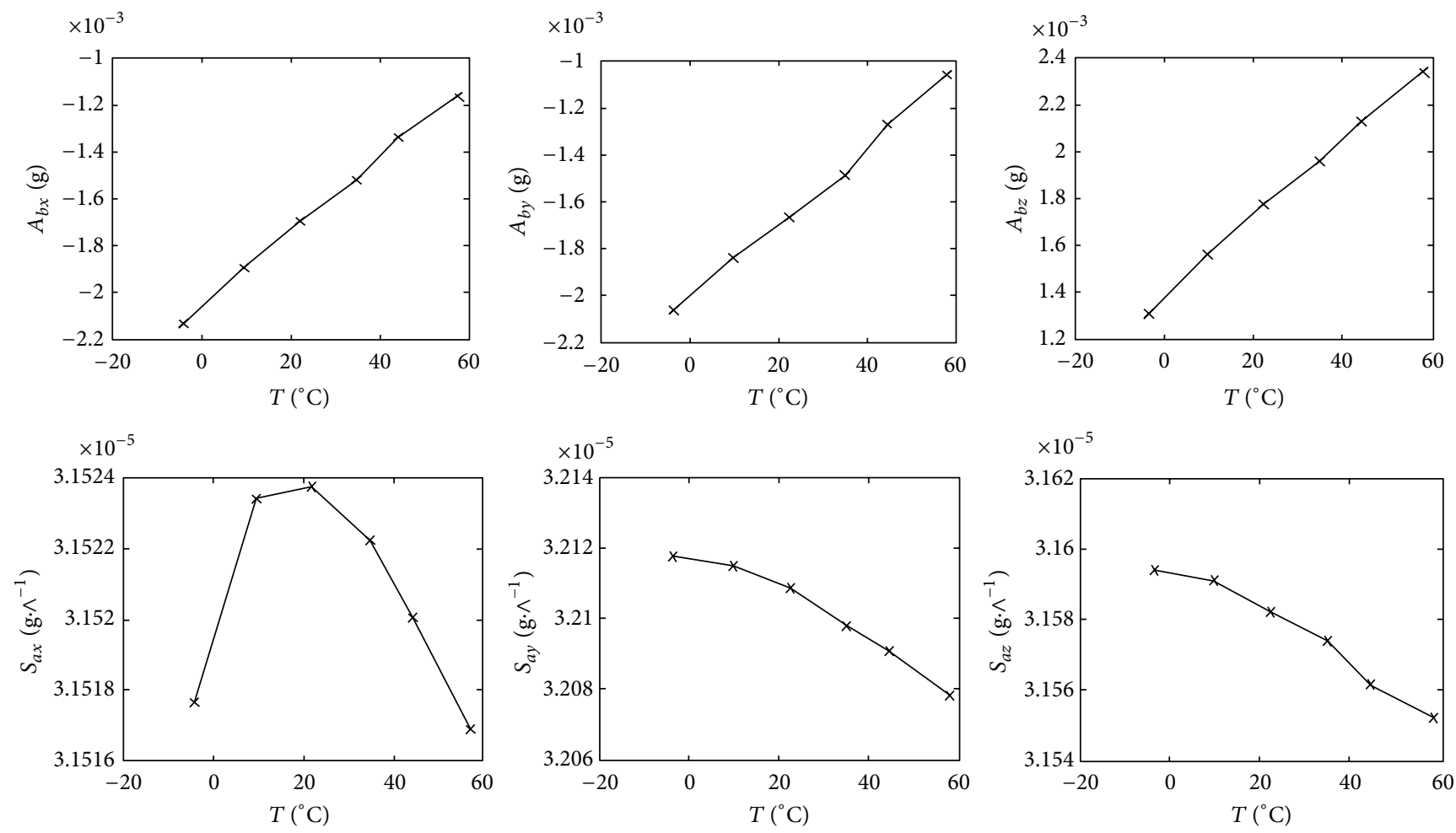

FIGURE 5: Curves of accelerometer bias and scale factor changing with temperature.

0.594 nautical mile/h from 1.850 nautical mile/h for vehicle experiment. The experiments validate the practicability and validity of the proposed method.

\section{Conclusion}

This paper proposed an optimization calibration method with four-direction rotations for RLG SINS based on a singleaxis turntable with temperature control and a high-precision hexahedral fixture in full temperature. Compared to the traditional 24-point dynamic-static hybrid calibration and linear fitting method, the inertial navigation average errors of the proposed method are reduced to 0.594 nautical mile/h from 1.850 nautical mile/h for vehicle experiments. The experimental results validate the practicability and validity of the proposed method.

\section{Acknowledgments}

The work was sponsored in part by the National Program on Key Basic Research Projects of China under Grant 2009CB724000, the CAST Innovation Foundation CAST201205, and in part by the National Natural Science Foundation of China under Grant nos. 60904093, 50975049, 60905056, 61004140, and 61273033.

\section{References}

[1] F. Jiancheng and Y. Sheng, "Study on innovation adaptive EKF for in-flight alignment of airborne POS," IEEE Transactions on
Instrumentation and Measurement, vol. 60, no. 4, pp. 1378-1388, 2011.

[2] Y. Yang and L. J. Miao, "Fiber-optic strapdown inertial system with sensing cluster continuous rotation," IEEE Transactions on Aerospace and Electronic Systems, vol. 40, no. 4, pp. 1173-1178, 2004.

[3] L. D. Zhang, J. X. Lian, M. P. Wu, and Z. Q. Zheng, "Research on auto compensation technique of strap-down inertial navigation systems," in Proceedings of the International Asia Conference on Informatics in Control, Automation, and Robotics (CAR '09), pp. 350-353, Bangkok, Thailand, September 2009.

[4] J. J. Hall and R. L. Williams, "Case study: inertial measurement unit calibration platform," Journal of Robotic Systems, vol. 17, no. 11, pp. 623-632, 2000.

[5] J. G. Lee, C. G. Park, and H. W. Park, "Multiposition alignment of strapdown inertial navigation system," IEEE Transactions on Aerospace and Electronic Systems, vol. 29, no. 4, pp. 1323-1328, 1993.

[6] B. Tadej, P. Janez, and M. Marko, “Three-axial accelerometer calibration using Kalman filter covariance matrix for online estimation of optimal sensor orientation," IEEE Transactions on Instrumentation and Measurement, vol. 61, no. 9, pp. 2501-2511, 2012.

[7] J. L. Li, J. C. Fang, and S. S. Ge, "Kinetics and design of a mechanically dithered ring laser gyroscope position and orientation system," IEEE Transactions on Instrumentation and Measurement, vol. 62, no. 1, pp. 210-220, 2013.

[8] F. Jiancheng and L. Jianli, "Integrated model and compensation of thermal errors of silicon microelectromechanical gyroscope," IEEE Transactions on Instrumentation and Measurement, vol. 58, no. 9, pp. 2923-2930, 2009. 
[9] A. Kim and M. F. Golnaraghi, "Initial calibration of an inertial measurement unit using an optical position tracking system," in Proceedings of the Position Location and Navigation Symposium (PLANS '04), pp. 96-101, April 2004.

[10] R. A. Chechile, A. H. Cherbettchian, and S. Spry, "Temperature compensated mount for supporting a ring laser gyro," US Patent 4890812, 1990.

[11] C. Shen and X. Y. Chen, "Analysis and modeling for fiber-optic gyroscope scale factor based on environment temperature," Applied Optics, vol. 51, no. 14, pp. 2541-2547, 2012. 


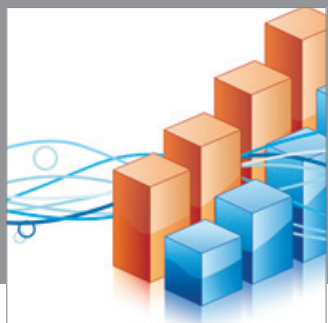

Advances in

Operations Research

mansans

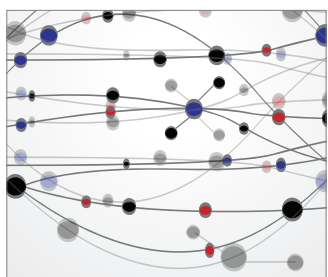

The Scientific World Journal
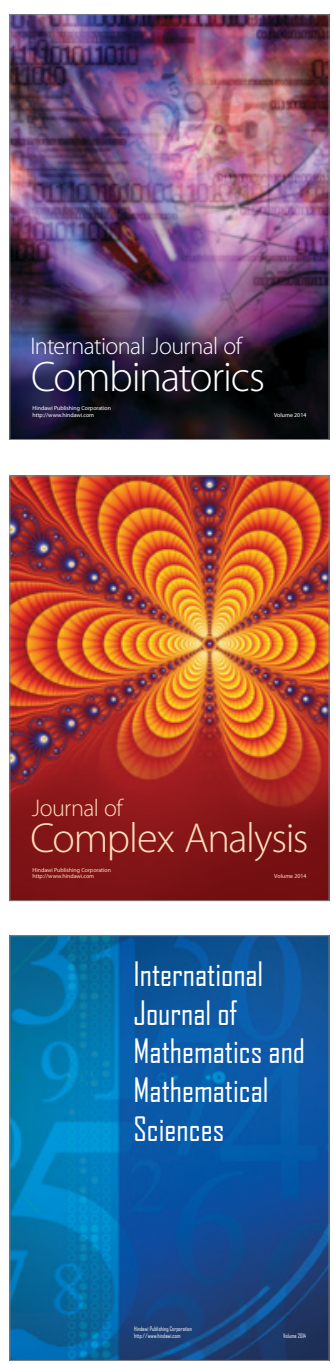
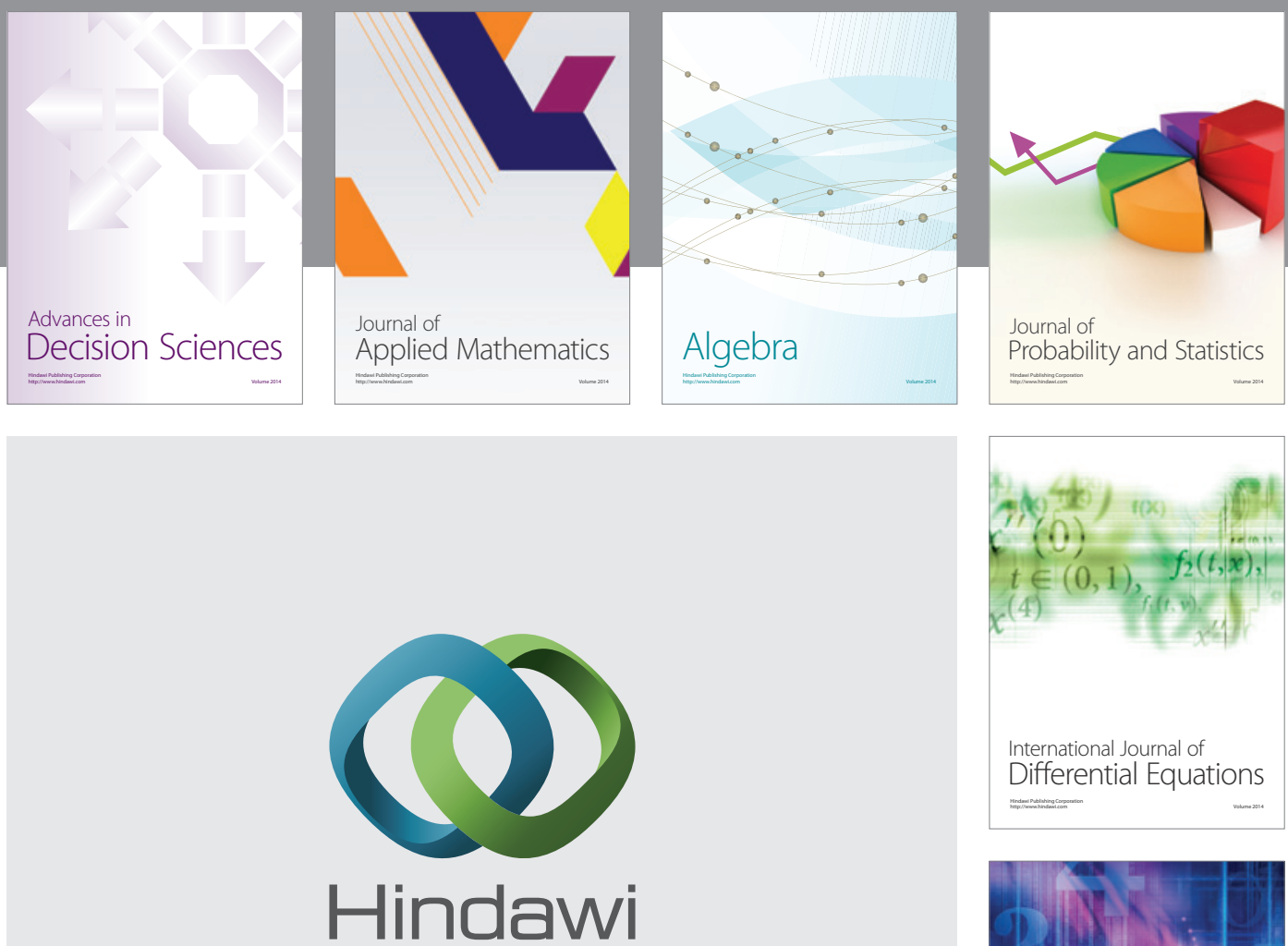

Submit your manuscripts at http://www.hindawi.com
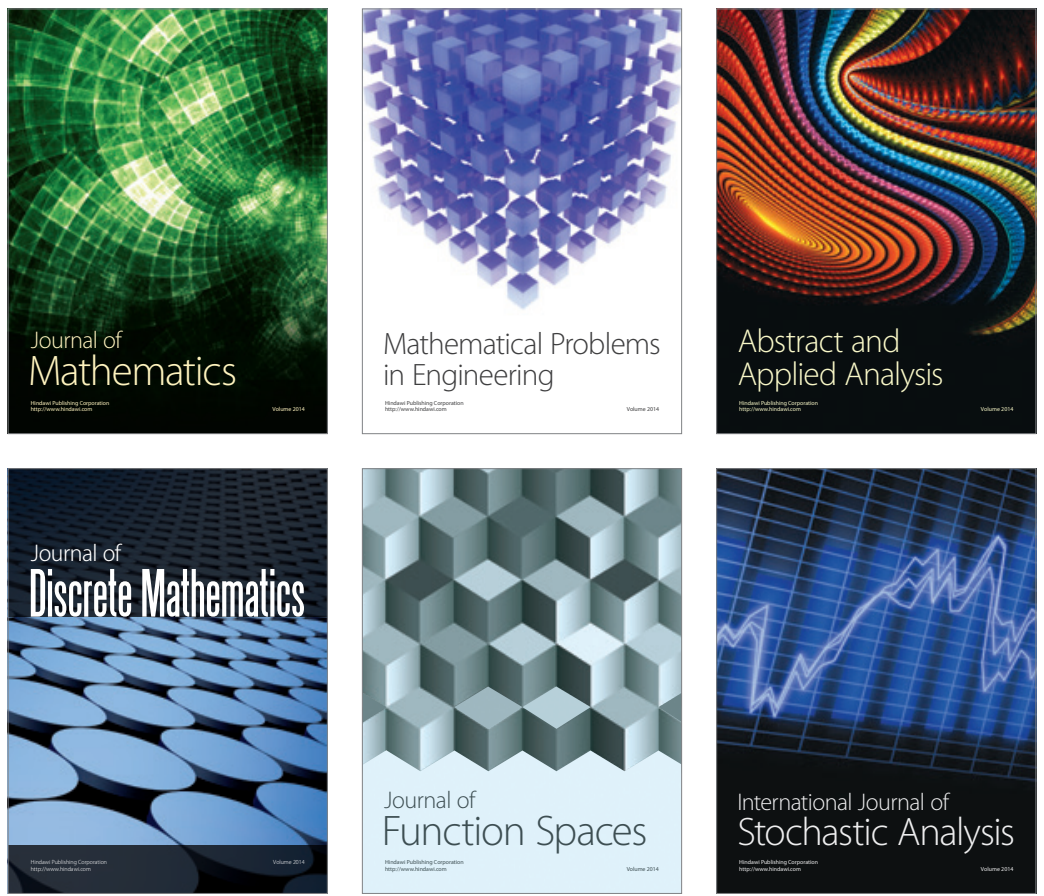

Journal of

Function Spaces

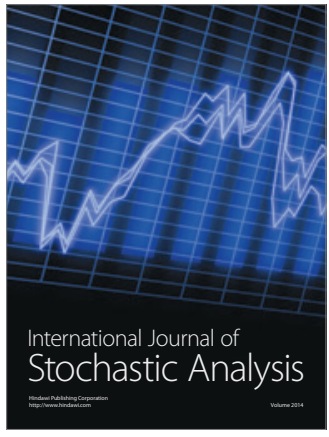

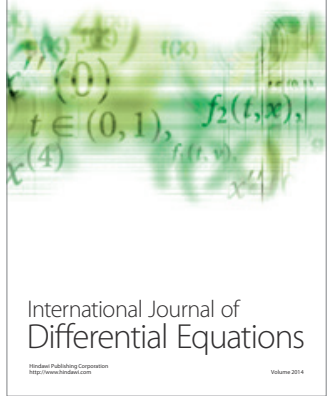
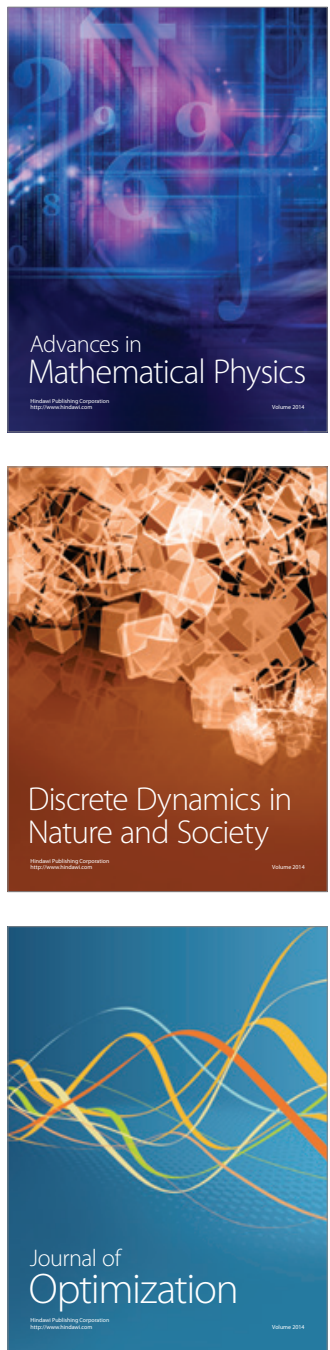